\title{
成蛋白: 一种新的细胞微丝骨架组装的调控因子
}

\author{
果春青 任海云 ${ }^{*}$ \\ (北京师范大学生命科学学院细胞生物学研究所, 细胞增殖与调控生物学教育部重点实验室, 北京 100875 . \\ *联系人, E-mail: hren@bnu.edu.cn)
}

\begin{abstract}
摘要 微丝骨架是真核细胞骨架的重要组成成分, 其动态结构在时空上受一系列肌动蛋白结合蛋白的 调节. 近年来，在众多的肌动蛋白结合蛋白中，成蛋白(formin)家族蛋白受到了更多的关注，因为它们 可以利用细胞内普遍存在的、与前纤维蛋白结合的肌动蛋白库形成不分支的微丝骨架结构. 目前, 来源 于不同生物的成蛋白家族成员已经得到大量报道, 对它们特性的认识亦愈加明确, 但是有一些问题尚 待阐明. 本文综述了近年来关于成蛋白家族蛋白在结构、功能和成核机理方面的研究进展, 并且比较了 该家族不同成员的理化特性, 以期为成蛋白的进一步深入研究提供更清晰的线索.
\end{abstract}

关键词 成蛋白 微丝骨架 肌动蛋白结合蛋白

在真核细胞中存在一个动态的微丝骨架系统, 其具有对各种发育和环境刺激信号反应的能力, 可 在聚合与解聚两种状态之间转变以调节细胞生长、分 裂和极性建立 ${ }^{[1,2]}$. 新的微丝可通过切割现有微丝形 成或者从单体肌动蛋白起始聚合形成 ${ }^{[3]}$. 由于球状 肌动蛋白的自发聚合不能满足微丝骨架的快速动态 变化, 所以需要肌动蛋白成核因子通过成核作用来 加速肌动蛋白聚合. 目前已知细胞内存在两类成核 因子, 即 Arp $2 / 3$ 复合体和成蛋白(formin). Arp $2 / 3$ 复合 体能促使形成微丝网络结构 ${ }^{[4]}$, 而成蛋白参与形成 不分支的微丝 ${ }^{[5]}$. 酵母、真菌、哺乳动物和植物中的 许多成蛋白基因或蛋白均已得到鉴定. 已知它们的 共同特征是都具有FH1 (formin homology 1)结构域和 FH2 (formin homology 2)结构域, FH1 结构域可与另 外一种重要的肌动蛋白结合蛋白一一前纤维蛋白 (profilin)相互作用, FH2 结构域则通过与肌动蛋白结 合发挥作用 ${ }^{[3]}$. 酵母、真菌和动物中的成蛋白已经得 到了深入的研究, 而对植物成蛋白研究的报道还十 分有限. 但是, 我们相信对植物成蛋白的研究必将使 我们对微丝骨架动态特性及调节的了解更加深入.

\section{1 成蛋白功能结构域和生化性质}

小鼠的limb deformity (ld) 基因是第一个研究的 成蛋白基因, 这个基因的突变导致小鼠不能形成 (“form”)正常的肢体和肾, 其因此得名 ${ }^{[6]}$. 此后, 来 自不同物种的成蛋白家族成员也相继被报道. 成蛋 白家族蛋白的显著特征是每一个物种的成蛋白都具 有多个成员. 例如, 在芽殖酵母(Saccharomyces cerevisiae)中有Bnilp和Bnr1p (Bni related) ${ }^{27}$; 裂殖酵母
(Schizosaccharomyces pombe)中有 Fus1, CDC12 和 For $3^{[8]}$; 在哺乳动物中发现了 15 个成蛋白同种型 (isoform), 包括mDia1 (mouse Diaphanous 1), mDia2, FRLs (formin related gene in leukocytes)等 [9]. 通过生 物信息学手段在植物中也发现了很多成蛋白家族成 员, 如在模式植物拟南芥中发现 22 个成员. 除此之 外, 在棉花、大豆、大麦、番茄、三叶草、苜宿、烟 草、水稻、宛豈、高粱、马铃薯、小麦、葡萄和玉米 中发现了 79 个可能的成蛋白相关基因. 值得关注的 是, 这些植物成蛋白可以划分为两类, 即 I 型和 II 型 [10]. 这些众多成员的存在可能暗示除了保守的成核 功能外, 成蛋白还具有很多其他的功能, 并且不同物 种的成蛋白和同一物种的不同成蛋白成员的功能也 可能存在差异.

成蛋白包含多个具有不同功能的结构域(图 1). 成蛋白的典型特征是其 C端都具有一个 FH (formin homology) 区域, 这是由 Castrillon和 Wasserman ${ }^{[14]}$ 首 次鉴定的. FH区域可进一步分为FH1 结构域、FH2 结 构域和连接它们的中间区域. 除FH结构域之外，不 同的成蛋白其 $\mathrm{N}$ 端或 $\mathrm{C}$ 端还具有其他不同的结构域, 如FH3 (formin homology 3)结构域、GBD (GTPase binding domain)、DAD (Dia-autoregulatory domain)、

BBS (Bud6p-binding site)、TM (trans-mem- brane domain)等 ${ }^{[8,15]}$. 研究表明, 不同的结构域赋予了成蛋 白不同的功能或者将成蛋白与其他信号分子相互联 系起来.

具有高度同源的 FH2 结构域是所有成蛋白成员 都具有的重要特征. FH2 结构域包含大约 400 个氨基 


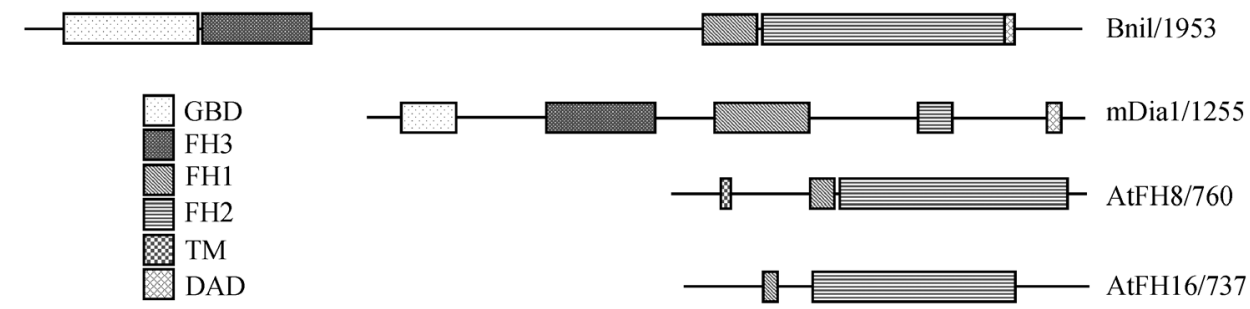

\begin{abstract}
200 aa
图 1 几个典型成蛋白成员的结构域组成

每一个成蛋白成员之后的数字显示了其氨基酸序列长度. 有关各个蛋白质的详细信息请参阅参考文献 [11 13]以及

http://kfrserver.natur.cuni.cz/genes/formin/index.html
\end{abstract}

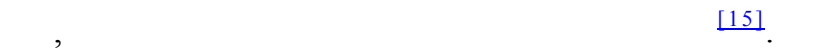
Bnilp的FH2 结构域晶体结构的研究表明，其可能以 二聚体的结构使肌动蛋白成核 ${ }^{[16,17]}$. FH1 结构域位于 $\mathrm{FH} 2$ 结构域的N端, 富含脯氨酸. 在许多情况下, FH1 结构域可与含有WW结构域、SH3 结构域的蛋白以及 前纤维蛋白相互作用 ${ }^{[15]}$. 但是并不是所有的成蛋白 都具有FH1 结构域, 例如Dictyostelium discoideum的 ForC和 21 个拟南芥成蛋白中的 4 个成员就缺失了 FH1 结构域 ${ }^{[8,18]}$. 有关成蛋白生化特性的研究多是基 于FH1FH2 结构域. 目前研究表明, 所有成蛋白的 FH1FH2 构建体都能起到成核作用，也能促进肌动蛋 白/前纤维蛋白复合体的聚合, 但是单独的FH2 结构 域只能促进肌动蛋白的聚合 $[5,11,19,20]$. 一旦肌动蛋白 开始聚合, FH1FH2 构建体会持续地与微丝的正端结 合, 这已经得到了电子显微镜观察的证实 ${ }^{[5]}$. 同时 Kovar等人 ${ }^{[211}$ 研究发现，成蛋白可以抑制微丝的相互 连接, 再次证明了这一结论. Higashida等人 ${ }^{[22]}$ 也在 活细胞中发现苂光标记的mDia可与延伸的微丝正端 连续地结合. 以上结果说明, 成蛋白的功能类似于封 端蛋白, 但是成蛋白作为封端蛋白对正端聚合的影 响是多样的. 小鼠的mDia对正端没 有任何影响 [23]. Bni1p FH1FH2 构建体可以减慢但不封闭正端延伸 ${ }^{[5]}$, 正端的聚合/解聚速率在存在Bni1p FH1FH2 时降低 $50 \%{ }^{[24]}$. CDC12p FH1FH2 构建体是一种典型的封端 蛋白, 它能抑制正端的聚合和解聚, 从而将 肌动蛋 白动态变化转移到负端 ${ }^{[21]}$. 成蛋白也能与微丝的侧 面结合并剪切微丝或者使微丝成束, 如单独的FRL1 和 $\mathrm{mDia} 2$ 的FH2 结构域就能使微丝成束 ${ }^{[25]}$, 小鼠的 成蛋白 FRL $\alpha$ 能够剪切微丝并且这也是其成核机制 的一部分 ${ }^{[23]}$. 但是并非所有的成蛋白都具有成束或 者剪切的功能, 如 m Dia 1 就不具有成束功
能 ${ }^{[25]}$.

在裂殖酵母成蛋白 Fus1 中还发现了 FH3 结构域, 它与自身的适当定位有关 ${ }^{[26]}$. GBD和DAD分别位于 Drfs (diaphanous-related formin)的 $\mathrm{N}$ 端和C端, 与Drfs 的活化有关 ${ }^{[27]}$. 但最近的研究表明, GBD可再分为与 自抑制有关的DID (diaphanous inhibitory domain)及与 RhoA结合有关的DID的N端序列 ${ }^{[28]}$.

植物成蛋白的结构域组成与其他生物的非常不 同, 如在拟南芥成蛋白的N端没有FH3, GBD或DAD, 其中一部分成员的 $\mathrm{N}$ 端存在跨膜结构域, 这类成员被 命名为 I 型成蛋白 $(\mathrm{AtFH} 1 \sim 11)$; 其他在 $\mathrm{N}$ 端不具有跨 膜结构域的成蛋白被命名为 II型成蛋白 (AtFH12 $21)^{[8]}$. 植物的成蛋白也显示了其他可变的结构域组 成. 基于这些结构域组成的差异可以将植物成蛋白 分成 $A \sim F$ 共 6 种类型 ${ }^{[10]}$. 这些结构差异可能暗示植物 成蛋白以另外的机制在与动物或真菌不同的信号通 路中发挥作用. 近年来, 已经有几个植物成蛋白的体 外生化性质得到研究. 与其他物种的成蛋白成员一 样, 目前研究的植物成蛋白 (FH1FH2)构建体(AtFH1, $\mathrm{AtFH} 4, \mathrm{AtFH} 5$ 和AtFH8)都具有成核功能并能促进形 成不分支的微丝, 并且FH2 结构域是成核的功能性 结构域 $[11,20,29,30]$. AtFHs (FH1FH2)构建体也与微丝正 端结合并以部分封端的方式改变正端的聚合与解聚 速率 [11,20,30]. AtFH1 的封端活性有一点特殊, 其 FH1FH2 构建体是一个“泄漏”的帽子, 只能部分地影 响正端的动态变化, 而FH2 构建体却是一个典型的 封端蛋白, 但只允许微丝在负端延伸 ${ }^{[20]}$. 植物成蛋 白的两个成员 $\mathrm{AtFH} 1^{[20]}$ 和AtFH8 (结果末显示)也具有 成束的功能, 并且AtFH8 FH1FH2 构建体也可剪切微 丝 $\frac{111]}{36}$. 


\section{2 成蛋白的晶体结构和成核机制}

目前对成蛋白成核机理的探究主要基于对其的 结构的分析与生化特性的测定. Bnilp FH2 结构域的 晶体结构表明, FH2 结构域形成了独特的“黏连二聚 体”, 从而将 2 个延伸的肌动蛋白头部䀿绑在一起. $\mathrm{Xu}$ 等人 ${ }^{[16]}$ 据此提出了“逐级行走”模型, 即黏连的二 聚体可能允许成蛋白在延伸中的正端上逐级行走, 并执行其成核和封端功能. “逐级行走”模型推测, FH2 同型二聚体的每一个部分只与一个肌动蛋白亚 基相互作用(一个与正端最后一个亚基相互作用，另 一个与倒数第二个亚基相互作用), 但是它不能解释 成蛋白如何保持并渐进地与快速延伸的正端结合以 及它如何随微丝旋转 ${ }^{[31]}$. Bnilp FH2 与TMR肌动蛋白 (四甲基罗丹明标记的肌动蛋白)的共结晶建立了一 个渐进移动模型(图 2). FH2 二聚体是稳定但却灵活

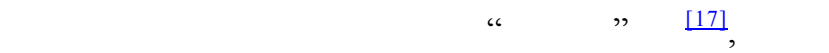
允许FH2 二聚体的每一个单体能同时与两个肌动蛋 白亚基相互作用. 因为在肌动蛋白亚基加入的过程 中只需要其中一个单体暂时脱离, 所以 $\mathrm{FH} 2$ 二聚体 能在肌动蛋白聚合过程中连续地与之结合 [31]. 在这 个模型中, FH2 二聚体以两种不同的状态存在, 即不 允许亚基加入的“闭合状态”和允许亚基加入的“开放 状态”. 在FH2 二聚体的滞后单位朝正端移动的过程 中这两种状态发生改变从而引起两个FH2 亚基功能

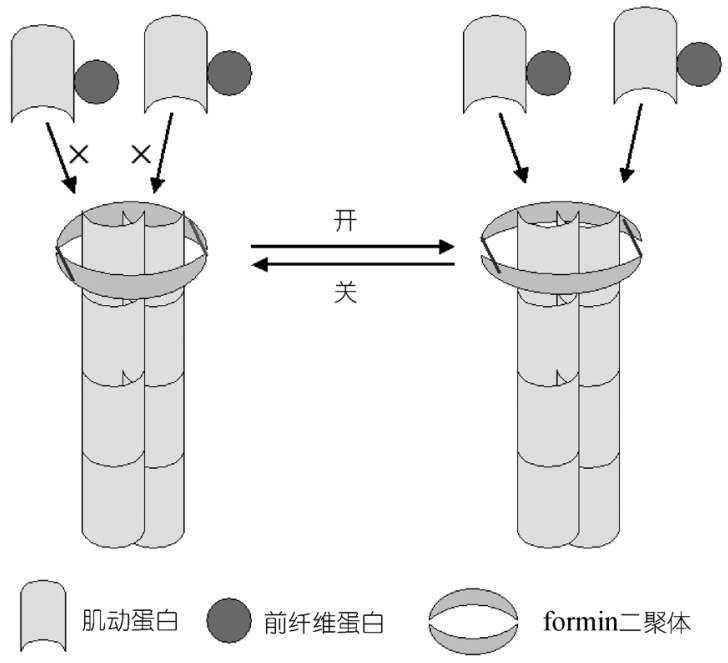

图 2 成蛋白成核以及封端活性的渐进移动模型 在这个模型中, 成蛋白形成了处于闭合/开放平衡之中的二聚体, 这个 二聚体围绕微丝的正端并渐进地与这个末端相结合. 当二聚体处于开 放状态时肌动蛋白/前纤维蛋白复合体可以加入. 不同的闭合/开放平 衡决定了各个成蛋白之间活性的差异. 更详细的描述请见参考文 献 [17,31]
的交换. 成蛋白中各种不同的闭合/开放平衡可能就 是不同成蛋白之间成核以及封端活性差异的原因 ${ }^{[17]}$.

\section{3 成蛋白的相互作用蛋白及其调节作用}

成蛋白是一种分子量较大的多结构域蛋白质, 因此, 除了肌动蛋白外, 它们还可与多种其他蛋白质, 如 Rho GTP 酶、前纤维蛋白、Src 类激酶和 eEF1A 等发生相互作用而受到调控, 以完成其调节微丝形 成的功能. 本文仅详细讨论两种研究较多的成蛋白 互作蛋白.

\subsection{Rho GTP 酶}

Rho GTP酶是是信号通路中的分子开关, 它通过 将GTP水解成GDP在“开”和“关”两种状态之间转变. 在对成蛋白的研究过程中, Drfs被鉴定为是Rho GTP 酶的受体，这类成蛋白的特征是其蛋白质的 N端具有 GBD, C端具有DAD. 活化的Rho GTP酶可与DAD竞 争结合GBD. 结合之后Drfs就可完成其在微丝聚合过 程中的功能 ${ }^{[15]}$. 已知能相互作用的Rho- 成蛋白包括 Bnilp-Rholp/Cdc42 ${ }^{[32]}$, Bnrlp-Rho3p/ Rho4p ${ }^{[7,33]}$, $\mathrm{mDia} 1-\mathrm{RhoA}^{[34]}$ 及 $\mathrm{mDia} 2-\mathrm{Cdc} 42^{[35]}$ 等. 当酵母或者哺 乳动物细胞中表达缺失 GBD的Drfs时会迅速诱导微 丝束的形成 ${ }^{[21]}$ ，表明Drfs位于Rho GTP酶信号通路的 下游. Li和Higgs ${ }^{[19]}$ 在体外证明了 $\mathrm{mDia} 1$ 的 $\mathrm{N}$ 端强烈地 抑制C端引起的肌动蛋白成核过程, 而RhoA能部分 地恢复这种抑制，但当与GDP或GTP类似物结合时不 能起到这种恢复作用. 因此, 影响Rho GTP酶的一些 因素可能通过Drfs对微丝骨架的影响执行其功能.

\section{2 前纤维蛋白}

前纤维蛋白是重要的单体肌动蛋白结合蛋白. 有关前纤维蛋白与成蛋白相互作用的信息主要来自 体外实验. 在成蛋白中, FH1 结构域是结合前纤维蛋 白所必需的. 最佳浓度的前纤维蛋白可加速正端延 伸, 如果将mDia FH1FH2 包被到聚苯乙烯珠子上形 成微丝后, 只有在前纤维蛋白存在的条件下肌动蛋 白才能添加到微丝的正端 ${ }^{[36]}$. 同时前纤维蛋白可使 被Cdc12 封闭的正端开始延伸 ${ }^{[21]}$. 对这些现象可以 这样解释: 首先, 前纤维蛋白与肌动蛋白的结合提高 了成蛋白二聚体的 $K_{\mathrm{O} / \mathrm{C}}$ (开放/闭合平衡); 其次, 前纤 维蛋白与肌动蛋白的结合帮助肌动蛋白朝向了更利 于添加到正端的方向, 并且与FH1 结合之后, 前纤维 蛋白在局部增加了前纤维蛋白-肌动蛋白的浓度 ${ }^{[31]}$. 体内实验也表明, - Bnilp (FH1FH2COOH)诱导微丝聚 
合的能力是依赖于前纤维蛋白的 [37]. 众所周知, 肌 动蛋白在体内与前纤维蛋白大约以 $1: 1$ 的比例存在, 所以前纤维蛋白与FH1 的相互作用对存在成蛋白条 件下肌动蛋白/前纤维蛋白复合体的聚合是必需的.

\section{3 植物成蛋白的相互作用蛋白}

因为在植物成蛋白中没有发现GBD, 所以似乎 它们不能与ROPs (Rho of plants)相互作用, 但是这种 假设还没有得到实验验证. 目前已用酵母双杂交方 法篮选出AtFH1 的相互作用蛋白FIP2 (formin interacting protein 2). 用大肠杆菌表达的重组蛋白进行的 实验表明, FIP2 能直接与 AtFH1 相互作用. FIP2 的氨 基酸序列与细菌推测的膜蛋白和动物的 $\mathrm{A}^{\text {型 }} \mathrm{K}^{+} \mathrm{ATP}$ 酶部分同源. AtFH1 可能与FIP2 形成膜针定的复合体 并参与微丝骨架的组织 ${ }^{[38]}$. 在体外pull down实验中 也观察到了前纤维蛋白与AtFH8 的相互作用 ${ }^{[11]}$. 此 外, Deeks等人 ${ }^{[29]}$ 利用酵母双杂交方法证明了 AtFH4 能与前纤维蛋白结合. 但是有关植物成蛋白相互作 用因子的信息还非常有限, 尚待深入研究.

\section{4 成蛋白的功能}

成蛋白可参与一系列与微丝骨架有关的生理活 动, 如细胞极性建立、细胞分裂和形态发生. 研究得 比较清楚的是酵母成蛋白的功能. Bnilp和Bnrlp 可以 在酵母中诱导微丝束的形成, 而在限制温度下生长 的bnr14bnil 4 - $t$ 双突变体则不能形成微丝束 ${ }^{[37]}$, 同 时Bnilp (FH1FH2)过量表达对酵母是致死的 ${ }^{[5]}$. BNII 基因突变之后能观察到极性分泌、mRNA定位、纺锤 体方向和细胞核位置方面的缺陷 $[37,39,40]$. 裂殖酵母 的 3 个成蛋白同种型Fus1, Cdc12 和For3 也参与了多 个与微丝相关的细胞活动: Fus 1 与结合过程中细胞顶 端的肌动蛋白极性有关 ${ }^{[26]} ; \mathrm{Cdc} 12$ 参与细胞分裂必需 的有丝分裂环的形成 ${ }^{[41]}$; For3 则参与对等细胞分裂 所需的微丝束的形成 ${ }^{[42]}$.

一些哺乳动物成蛋白同种型参与了与微丝骨架 相关的生理活动. 小鼠的formin-1 是第一个被鉴定的 成蛋白, 它参与细胞信号转导以及肢体和肾脏的形 态发生 ${ }^{[6]} \mathrm{mDia} 1$ 和 $\mathrm{mDia} 2$ 为 2 个参与应力纤维形成 和SRF (血清反应因子)活化的小鼠成蛋白 ${ }^{[43,44]}$. 人的 Fhos与脾脏的形态发生有关 ${ }^{[45]}$. 活化的Fhos 2 及

Fhos 1 (两种Fhos同种型)的过量表达在HeLa细胞中诱 导肌动蛋白应力纤维的形成. Fhos2 也可与nestin结合, 这可能暗示Fhos 2 介导了 nestin中间纤维和微丝之间
的联系 ${ }^{[46]}$.

在植物成蛋白家族中, 一些拟南芥成蛋白同种 型的功能已经得到研究, 其中包括 AtFH1, AtFH5, AtFH6 和AtFH8. AtFH1 为第一个研究体内功能的植 物成蛋白. AtFH1 在花粉中的过量表达会诱导形成微 丝束阵列, 并且这些阵列是从细胞膜开始指向细胞 质的. AtFH1 表达的轻度增加能刺激生长, 但是过量 表达会导致花粉管变宽, 生长失去极性及生长的停 止. 这些结果表明, AtFH1 调节的肌动蛋白聚合对花 粉细胞的极性生长过程非常重要 ${ }^{[47]}$. Ingouff等人 ${ }^{[30]}$ 发现, AtFH5 功能的丧失干扰了胚乳后孔的适当形态 发生, 并且AtFH5-GFP融合蛋白会在细胞分裂所需 的细胞板位置累积. AtFH6 由Favery等人 ${ }^{[48]}$ 用启动子 捕获策略分离. 在巨大细胞的形成过程中, AtFH6 被 上调表达并且均匀分布于质膜的位置. 此外, AtFH6 可以抑制酿酒酵母bnr1 0 bni $1 \Delta$ 突变体的出芽缺陷, 表 明AtFH6 调节由微丝束聚合控制的极性生长. 我们 对 AtFH8 过量表达株系的研究发现, 高水平的AtFH8 可以引起异常的根毛表型, 这包括波浪状的根毛、顶 端膨大的根毛、不同程度分支的根毛以及在一个生根 毛位置长出两个根毛, 这些异常的根毛代表了细胞 极性的丧失. 与异常的根毛表型伴随的是这些根毛 中微丝骨架分布的异常, 表明这是AtFH8 调节的微 丝骨架相关的过程 ${ }^{[11]}$. Deeks等人 ${ }^{[29]}$ 有关AtFH8 的工 作显示了 AtFH8 影响根和根毛的发育. 植物成蛋白 中的所有发现提示, AtFHs也在植物的极性、形态发生 和细胞分裂中发挥重要作用.

对酵母和哺乳动物成蛋白的研究中还发现, 它 们还可以影响微管的组织形成. 在酵母中, 肌球蛋白 V (Myo2p)控制纺锤体的方向, 它将微管顶端复合体 (MT tip complex)递送到芽的部位. Bnilp诱导聚合的 微丝束为Myo2p提供了轨道 ${ }^{[15]}$. 所以, Bnilp间接地 影响了出芽过程中的极性. 在哺乳动物细胞中, $\mathrm{mDia}$ 也与微管的组织有关, $\mathrm{mDia} 1$ 和 $\mathrm{mDia} 2$ 在体内与微管 阵列共定位 ${ }^{[49,50]}$, 同时它们还可以在血清饥饿的 NIH $3 T 3$ 细胞中诱导稳定的微管 ${ }^{[49]}$. mDia在微管组织中 的作用还需要进一步阐明. 但是目前还没有任何关 于植物成蛋白与微管相互作用的报道.

\section{5 展望}

成蛋白是分子量较大的多结构域蛋白, 能使前 1 纤维蛋白/肌动蛋白复合体在正端聚合并形成长的不 
分支的微丝束. 由于成蛋白在正端的封端作用，所形 成的微丝束可达到很长的长度. 有关成蛋白的生化 性质已经得到深入的研究，它们都具有成核以及封 端的活性，但不同成员之间仍存在差异. 晶体结构的 研究表明成蛋白的成核机理是保守的, 即以二聚体 的方式发挥作用. 然而, 成核的机理仍有待进一步研 究, 因为还未获得全长成蛋白的晶体结构. 与酵母和 动物不同的是, 我们对于植物成蛋白的了解还很少. 目前只有部分的 I 型成蛋白得到研究, 对于 II 型成 蛋白还一无所知. 我们还不了解植物成蛋白以什么 样的机制发挥作用, 这种机制是否与酵母或动物的 相同或者不同，而且还没有提出一个植物成蛋白的 工作模型. 这一问题之所以重要, 是因为人们预测植 物成蛋白可能在与动物和酵母不同的信号通路中发 挥重要作用 ${ }^{[8]}$. 因此, 还有很多问题尚待阐明: 不同 成员之间在体内和体外功能上是否有差异? 植物成 蛋白活性是如何被调节的? 它们在信号通路中处于 什么样的位置? 这些问题的解释必将使我们对成蛋 白以及对微丝骨架的理解迈入更深的一步.

致谢本工作为国家杰出青年科学基金(批准号: 30325005)、国家自然科学基金(批准号: 30470176)和国家 重点基础研究发展计划(批准号: 2006CB100100)资助项目.

\section{参考文献}

1 徐霞, 些惠君, 孙一娜, 等. 花粉原生质体极性重建及萌发过程 中的微丝骨架列阵. 科学通报, 2004, 49(15): 1507-1511

2 陈忠才, 蔡尚, 蒋青, 等. 微管和微丝骨架综合调控动物细胞胞 质分裂过程. 科学通报, 2005, 50(3): 225一-231

3 Evangelista M, Zigmond S, Boone C. Formins: Signaling effectors for assembly and polarization of actin filaments. J Cell Sci, 2003, 116: 2603-2611[DOI]

4 Higgs H N, Pollard T D. Regulation of actin filament network formation through Arp2/3 complex: Activation by a diverse array of proteins. Annu Rev Biochem, 2001, 70: 649—676[DOI]

5 Pruyne D, Evangelista M, Yang C, et al. Role of formins in actin assembly: Nucleation and barbed-end association. Science, 2002, 297: 612-615[DOI]

6 Kleinebrecht J, Selow J, Winkler W. The mouse mutant limbdeformity (ld). Anat Anz, 1982, 152: 313-324

7 Imamura H, Tanaka K, Hihara T, et al. Bnilp and Bnrlp: Downstream targets of the Rho family small G-proteins which interact with profiling and regulate actin cytoskeleton in Saccharomyces cerevisiae. EMBO J, 1997, 16: 2745-2755[DOI]

8 Deeks M J, Hussey P J, Davies B. Formins: Intermediates in signal-transduction cascades that affect cytoskeletal reorganization. Trends Plant Sci, 2002, 7: 492-498[DOI]

9 Higgs H N, Peterson K J. Phylogenetic analysis of the formin ho-

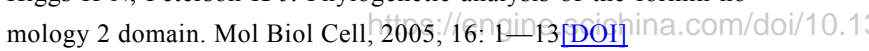

10 Cvrčková F, Novotný M, Pícková D, et al. Formin homology 2 domains occur in multiple contexts in angiosperms. BMC Genomics, 2004, 5: 1471-2164

11 Yi K X, Guo C Q, Chen D, et al. Cloning and functional characterization of a formin-like protein (AtFH8) from Arabidopsis. Plant Physiol, 2005, 138: 1071-1082[DOI]

12 Moseley J B, Sagot I, Manning A L, et al. A conserved mechanism for Bni1- and mDial-induced actin assembly and dual regulation of Bni1 by Bud6 and profilin. Mol Biol Cell, 2004, 15: 896907[DOI]

13 Krebs A, Rothkegel M, Klar M, et al. Characterization of functional domains of mDia1, a link between the small GTPase Rho and the actin cytoskeleton. J Cell Sci, 2001, 114: 3663-3672

14 Castrillon D H, Wasserman S A. Diaphanous is required for cytokinesis in Drosophila and shares domains of similarity with the products of the limb deformity gene. Development, 1994, 120: $3367-3377$

15 Wallar B J, Alberts A S. The formins: Active scaffolds that remodel the cytoskeleton. Trends Cell Biol, 2003, 13: 435-446[DOI]

$16 \mathrm{Xu} \mathrm{Y,} \mathrm{Moseley} \mathrm{J} \mathrm{B,} \mathrm{Sagot} \mathrm{I,} \mathrm{et} \mathrm{al.} \mathrm{Crystal} \mathrm{structures} \mathrm{of} \mathrm{a} \mathrm{formin}$ homology-2 domain reveal a tethered dimer architecture. Cell, 2004, 116: 711-723[DOI]

17 Otomo T, Tomchick D R, Otomo C, et al. Structural basis of actin filament nucleation and processive capping by a formin homology 2 domain. Nature, 2005, 433: 488-494[DOI]

18 Kitayama C, Uyeda T Q. ForC, a novel type of forming family protein lacking an FH1 domain, is involved in multicellular development in Dictyostelium. J Cell Sci, 2003, 116: 711-723[DOI]

19 Li F, Higgs $\mathrm{H} \mathrm{N}$. The mouse formin mDial is a potent actin nucleation factor regulated by auto inhibition. Curr Biol, 2003, 13: 1335-1340[DOI]

20 Michelot A, Guérin C, Huang S J, et al. The formin homology 1 domain modulates the actin nucleation and bundling activity of Arabidopsis FORMIN1. Plant Cell, 2005, 17: 2296-2313[DOI]

21 Kovar D R, Kuhn J R, Tichy A L, et al. The fission yeast cytokinesis formin $\mathrm{Cdc} 12 \mathrm{p}$ is a barbed end actin filament capping protein gated by profilin. J Cell Biol, 2003, 161: 875-887[DOI]

22 Higashida C, Miyoshi T, Fujita A, et al. Actin polymerization-driven molecular movement of mDia1 in living cells. Science, 2004, 303: 2007-2010[DOI]

23 Harris E S, Li F, Higgs H N. The mouse formin, FRL $\alpha$, slows actin filament barbed end elongation, competes with capping protein, accelerates polymerization from monomers, and severs filaments. J Biol Chem, 2004, 279: 20076-20087[DOI]

24 Pring M, Evangelista M, Boon C, et al. Mechanism of formin- induced nucleation of actin filaments. Biochemistry, 2003, 42: 486496[DOI]

25 Harris E S, Rouiller I, Hanein D, et al. Mechanistic differences in actin bundling activity of two mammalian formins, FRL1 and mDia2. J Biol Chem, 2006, 281: 14383-14392[DOI]

26 Petersen J, Nielsen O, Egel R, et al. FH3, a domain found in formins, targets the fission yeast formin Fus 1 to the projection tip during conjugation. J Cell Biol, 1998, 141: 1217-1228[DOI] 
27 Alberts A S. Identification of a carboxyl-terminal Diaphanous-related forming homology protein autoregulatory domain. J Biol Chem, 2001, 276: 2824-2830[DOI]

28 Li F, Higgs H N. Dissecting requirements for auto-inhibition of actin nucleation by the formin, mDia1. J Biol Chem, 2005, 280: 6986-6992[DOI]

29 Deeks M J, Cvrcková F, Machesky L M, et al. Arabidopsis group Ie formins localize to specific cell membrane domains, interact with actin-binding proteins and cause defects in cell expansion upon aberrant expression. New Phytologist, 2005, 168: 529$540[\mathrm{DOI}]$

30 Ingouff M, Gerald J N F, Guérin C, et al. Plant formin AtFH5 is an evolutionarily conserved actin nucleator involved in cytokinesis. Nat Cell Biol, 2005, 7: 374-380[DOI]

31 Kovar D R. Molecular details of formin-mediated actin assembly. Curr Opin Cell Biol, 2006, 18: 11-17[DOI]

32 Evangelista $\mathrm{M}$, Blundell K, Longtine $\mathrm{M} \mathrm{S}$, et al. Bnilp, a yeast formin linking cdc42p and the actin cytoskeleton during polarized morphogenesis. Science, 1997, 276: 118-122[DOI]

33 Robinson N G, Guo L, Imai J, et al. Rho3 of Saccharomyces cerevisiae, which regulates the actin cytoskeleton and exocytosis, is a GTPase which interacts with Myo2 and Exo70. Mol Cell Biol, 1999, 19: 3580-3587

34 Watanabe N, Madaule P, Reid T, et al. p140mDia, a mammalian homolog of Drosophila diaphanous, is a target protein for Rho small GTPase and is a ligand for profilin. EMBO J, 1997, 16: 3044 -3056[DOI]

35 Peng J, Wallar B J, Flanders A, et al. Disruption of the diaphanous-related formin Drf1 gene encoding mDial reveals a role for Drf3 as an effector for Cdc42. Curr Biol, 2003, 13: $534-$ $545[\mathrm{DOI}]$

36 Romero S, Le Clainche C, Didry D, et al. Formin is a processive motor that requires profilin to accelerate actin assembly and associated ATP hydrolysis. Cell, 2004, 119: 419—429[DOI]

37 Evangelista M, Pruyne D, Amberg D C, et al. Formins direct Arp2/3-independent actin filament assembly to polarize cell growth in yeast. Nat Cell Biol, 2002, 4: 32-41[DOI]

38 Banno H, Chua N H. Characterization of the Arabidopsis formin-like protein $\mathrm{AFH} 1$ and its interacting protein. Plant Cell Physiol, 2000, 41(5): 617-626
39 Fujiwara $\mathrm{T}$, Tanaka $\mathrm{K}$, Inoue $\mathrm{E}$, et al. Bnilp regulates microtubule-dependent nuclear migration through the actin cytoskeleton in Saccharomyces cerevisiae. Mol Cell Biol, 1999, 19: $8016-8027$

40 Lee L, Klee S K, Evangelista M, et al. Control of mitotic spindle position by the Saccharomyces cerevisiae formin Bnilp. J Cell Biol, 1999, 144: 947-961[DOI]

41 Chang F, Drubin D, Nurse P, et al. Cdc12p, a protein required for cytokinesis in fission yeast, is a component of the cell division ring and interacts with profilin. J Cell Biol, 1997, 137: 169-182[DOI]

42 Feierbach B and Chang F Roles of the fission yeast formin For3p in cell polarity, actin cable formation and symmetric cell division. Curr Biol, 2001, 11: 1656-1665[DOI]

43 Copeland J W, Treisman R. The Diaphanous-related formin mDia1 controls serum response factor activity through its effects on actin polymerization. Mol Biol Cell, 2002, 13: 4088 - 4099[DOI]

44 Tominaga T, Sahai E, Chardin P, et al. Diaphanous-related formins bridge Rho GTPase and Src tyrosine kinase signaling. Mol Cell, 2000, 5: 13-25[DOI]

45 Westendorf J J. The formin/diaphanous-related protein, FHOS, interacts with Rac1 and activates transcription from the serum response element. J Biol Chem 2001, 276: 46453 - 46459[DOI]

46 Kanaya H, Takeya R, Takeuchi $\mathrm{K}$, et al. Fhos2, a novel formin-related actin-organizing protein, probably associates with the nestin intermediate filament. Genes Cells, 2005, 10: 665678[DOI]

47 Cheung A Y, Wu H M. Overexpression of an Arabidopsis formin stimulates supernumerary actin cable formation from pollen tube cell membrane. Plant Cell, 2004, 16: 257-269[DOI]

48 Favery B, Chelysheva L A, Lebris M, et al. Arabidopsis formin AtFH6 is a plasma membrane-associated protein upregulated in giant cells induced by parasitic nematodes. Plant Cell, 2004, 16: 2529-2540[DOI]

49 Palazzo A F, Cook T A, Alberts A S, et al. mDia mediates Rho-regulated formation and orientation of stable microtubules. Nat Cell Biol, 2001, 3: 723-729[DOI]

50 Kato T, Watanabe N, Morishima Y, et al. Localization of a mammalian homolog of diaphanous, mDial, to the mitotic spindle in HeLa cells. J Cell Sci, 2001, 114: 775-778

(2006-06-29 收稿, 2006-09-04 接受) 